\title{
RADIOACTIVE EVENT BIFURCATION - AN EXAMPLE OF DISTRIBUTION
}

\author{
Celso Luis Levada \\ Huemerson Maceti \\ Ivan J. Lautenschleguer \\ FUNDAÇÃO HERMINIO OMETTO UNIARARAS - BRAZIL \\ celsolevada@yahoo.com.br
}

\section{ABSTRACT}

The present text contains a proposal to analyze the behavior of a beam of particles that, due to some physical mechanism, is divided into two. For the purpose of clarification, we emphasize that the term to be used to designate the process that has occurred will be bifurcation.

This fact, we inform that the pretension of this article is to discuss probabilistically the effect of the bifurcation of the beam establishing a distribution function with calculations of its standard and average value.

The expectation is also to be able to discuss the characteristics of the function and analyze, mainly its average value, as a function of the average number of incidents and suppressed events. It is intended to direct the analysis, preferably, towards a possible comparison with phenomena reported in the literature, notably facts that are linked to possible distortions caused by the measuring system, supposedly mounted by an observer, in order to monitor the bifurcation mechanism.

\section{Keywords}

bifurcation, nuclear disintegration, Lommel functions

\section{INTRODUCTION}

A typical case of partitioning of a radiation beam is observed when a screen with two slits between the meter and the source is inserting. The presence of the slots offers two possibilities of access to the meter, presenting, in this way, as a bifurcation mechanism.

It is to be announced that the purpose of the mention made to the double-slit device is, initially, to exemplify a physical device which deflects a beam in two directions. In fact, the specialized literature (1) reports this issue well, with respect to possible anomalies caused by the presence of the measuring instrument. Thus, moved by this intention, we will continue with a few more quotations.

A description of the procedure adopted to cause unfolding in photon bundles is identified in reference (2).

A beam is projected onto a semi-mirrored mirror that sends it to two converters. During the operation the beam is divided into two others, by a splitting device, and then another arrangement rejoins the beams, directing it to a detector.

A source sends pairs of photons in opposite directions, which are bifurcated and destined to two detectors, in order to an evaluation of possible correlations between the measurements, a subject that can be better understood, using the observations of Aspect ${ }^{(3)}$

The experiment can be carried out with the incidence of proton pairs on filters and detectors, however, the authors $^{(2)}$ suggest the use of specially prepared carbon slides in order to provide the scattering of the incident protons. This procedure resembles the bifurcation process.

In this regard, Plaga ${ }^{(8)}$ develops an interesting study on interactions of distant particles, while Bennette, cited in reference (4) suggest experiments to practice such correlations, in which two observers A and B, with linear and circular polarizers, emit a photon sequence whose polarization is chosen randomly.

The illustration in Figure 1 is very suggestive, in the sense of providing a visual effect of the experiment involving event bifurcation.

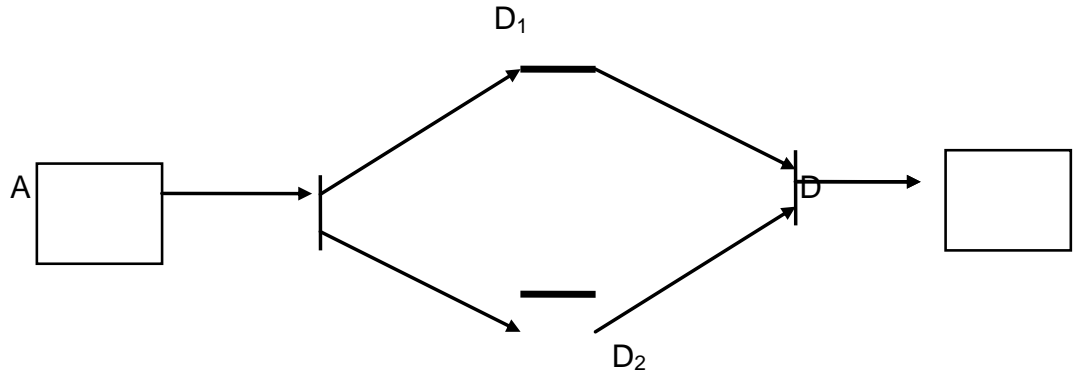

Figure 1 - Unfolding and reunification of a beam 


\section{POISSON DISTRIBUTION}

The nuclear disintegration process is governed by the Poisson distribution, as demonstrated by Bateman ${ }^{(10)}$ at the beginning of the century. The subject was thoroughly discussed and analyzed by numerous researchers, among them those mentioned in references (5) and (6).

In fact, not only this process, but others, including those from the count of photons of conventional light, as can be seen in reference (7), where we can observe the existence of a Poisson statistic for light waves. The author also distinguishes between Poisson statistic associated with coherent light, laser type, for example, of common light that obeys a super Poisson statistic. Other optical effects produce sub Poisson light.

In fact, we say that a set of particles obeys the Poisson distribution, when it fits into a function of the type

$$
P_{n}(E)=\frac{E^{n}}{n !} e^{-E}
$$

The parameter $E$ corresponds to the average number of events recorded during a time interval $t$, when the mean time between two consecutive events is regarded. Clarifying, if we fix a given time, $t$, and ask: what is the number of disintegrations of the radioactive sample? We will answer any integer, $\mathrm{n}$, from zero to infinity. Of course some values are more likely. In fact, the one with the highest probability is exactly the number $\mathrm{n}$ that coincides, or at least a lot approaches the mean number $\mathrm{E}$.

\section{BIFURCATION STUDY}

According to the model initially proposed, that is, separation of the beam into two parts, shown in figure 1 , it is noticed that a combination of probabilities occurs, that is, there is a double probabilistic effect.

Given the sample and a bifurcation there is, first, the probability of occurrence of, say, $(m+n)$ nuclear disintegration events, at a given location and instant. Then, by offering two choice options for the trajectory of the formed particles, we may have the deviation of $m$ of them to the right as well as of $n$ to the left.

In fact, it is a composition of probabilities where, initially, the Poisson law governs the $(m+n)$ events of the disintegration and, once the beam is divided, there will be a law for the regency of suppressed events considered, by hypothesis, which obeys the Poisson law.

Suppose a radioactive source emits a particle beam, directed to a counter identified by the symbol $\mathrm{A}$.

A second counter, designated by the symbol $B$, will be available to "receive" the portion of the beam deviated from the direction of $A$.

In order to provide a visualization of the experience, we will propose a schematic diagram, identified by figure 2.

According to the illustration of figure 2, the observations and control will be performed from the point $\mathrm{O}$, in the register counter. Another counter, identified as suppression, will be represented by the symbol $Q$.

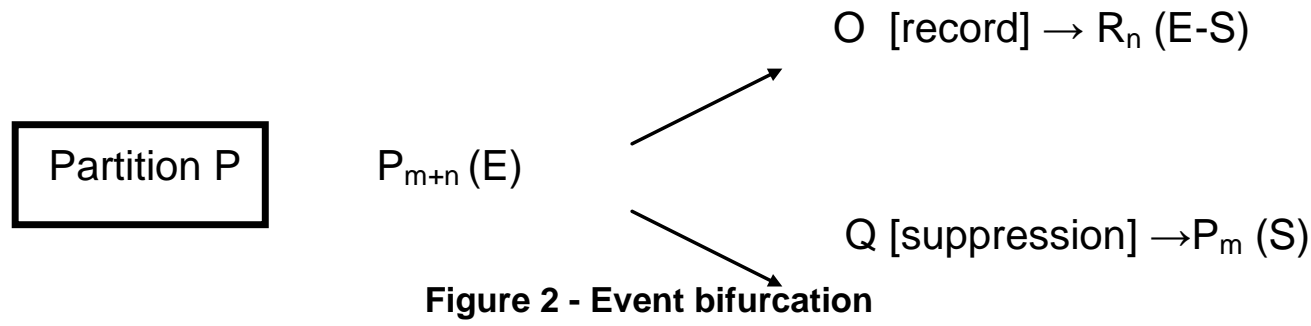

In this case, the fate of the incident events will be decided through two possibilities:

$\mathrm{a}_{1}$ ) Passing through the counting channel of counter $A$, monitored by an observer $A$

$\mathrm{a}_{2}$ ) Passing through the suppression channel, by means of a counter $B$, inspected by an observer $B$.

For the purposes of calculations, we will designate:

$E$, Average number of incident events

$R$, Average number of events recorded on channel A

$S$, Average number of events suppressed (from A), addressed to B 
Moreover, having the information, or at least the intuition that, with separation, the beam will retain its essence of being radioactive, it is quite possible that they will remain obeying the Poisson distribution. Some observer, perhaps with curiosity, may wish to test such a hypothesis.

By observing the bundles after the bifurcation and, knowing that they originate from a radioactive sample, we can assume that they obey Poisson distribution.

In this case, the position to be taken should be to write the probability distribution from one of the possible points of observation, $\mathrm{O}$ or $\mathrm{Q}$, strategically installed, that is, at the back in relation to the partition.

\section{SEQUENCE OF POSSIBILITIES}

In other words, we can say that there are $(m+n)$ photons that obey Poisson law focusing on the point $P$, from which photons, also governed by Poisson law, emerge towards the point $\mathrm{Q}$ whereas $\mathrm{n}$ of them they orient themselves towards the sector $\mathrm{O}$. That is, the observer, in $\mathrm{O}$, asks: If we have a counter, what is the distribution of $\mathrm{n}$ events, $\mathrm{Rn}$, to be recorded?

In order to answer the question, the procedure to be followed must be identical to the one executed in the previous paragraph, whose essence is emphasized in the following illustrative example, where will be considered the possibility of registering, say, five events.

The probable sequence, associated to an occurrence of five records in $\mathrm{O}$, will be: five incidences and no suppression, or, six incidences and one suppression, or, seven incidences and two deletions ... etc.

By the way, it should be noted that, for simplifications, the deviation of part of the beam to the point $Q$ is being considered as suppression.

Thus, supposing that $E$ and $S$ are, respectively, the mean number of events incident and suppressed and representing this probability by $R_{5}$, we can write

$$
R_{5}=\sum_{m=0}^{\infty} P_{m+5}(E) P_{m}(S)
$$

In the case of a generic number, $\mathrm{n}$, of records, we will have

$$
R_{n}=\sum_{m=0}^{\infty} P_{m+n}(E) P_{m}(S)
$$

In accordance with the definition given in (1) and, in view of the fact that the Bessel functions obey the form

$$
\begin{array}{r}
I_{n}(x)=\sum_{j=0}^{\infty}(x / 2)^{n+2 j} /(n+j) ! j ! \\
\text { it can be verified that } R_{n}=I_{n}(x) h^{n} e^{-(E+S)}
\end{array}
$$

being that

$$
h=\sqrt{\frac{E}{S}} \quad \text { e } x=2 \sqrt{E . S}
$$

From what has been found, the distribution of records thus understood follows a Bessel distribution. 
By definition the norm of a function is given by :

$$
N\left(R_{n}\right)=\sum_{n=0}^{\infty} h^{n} I_{n}(x) \cdot e^{-(E+S)}
$$

Using the fact that $I_{n}(x)=i^{-n} J_{n}(i . x)$, and considering the Lommel functions defined by

$$
\begin{aligned}
U_{n}(y, x) & =\sum_{m=0}^{\infty}(-1)^{m} \cdot\left(\frac{y}{x}\right)^{n+2 m} \cdot J_{n+2 m}(x) \\
h & =\sqrt{\frac{E}{S}} \text { e } x=2 \sqrt{E . S}
\end{aligned}
$$

it can be said that if the series observed in the function $\mathrm{Rn}(\mathrm{x})$ is absolutely convergent, that is, converges into a module, we can use the following theorem: "Regrouping the terms of an absolutely convergent series, the new sequence obtained also converges to the same value."

$$
N\left(R_{n}\right)=e^{-(E+S)}\left\{\sum_{n=0}^{\infty}[-i h]^{2 n} \cdot J_{2 n}(2 i x)+\sum_{n=0}^{\infty}[-i h]^{2 n+1} J_{2 n+1}(2 i x)\right\}
$$

For reasons of comparison with the Lommel functions, we obtain the following transformation

$$
\frac{E}{S}=\frac{2 i E}{2 i \sqrt{E S}}
$$

Thus it is possible to write the norm of the distribution function $N(R n)$ as

$$
e^{-(E+S)} \cdot\left\{\sum_{n=0}^{\infty}(-i)^{2 n}\left[\frac{2 i E}{2 i \sqrt{E \cdot S}}\right]^{2 n} \cdot J_{2 n}\left(2 i \sqrt{E S}+\sum_{n=0}^{\infty}(-i)^{2 n+1}\left[\frac{2 i E}{2 i \sqrt{E S}}\right]^{2 n+1} \cdot J_{2 n+1}(2 i \sqrt{E S})\right\}\right.
$$

Introducing the Lommel functions of zero and one indexes, it comes that

$$
N\left(R_{n}\right)=e^{-(E+S)}\left\{\left[U_{0}(2 i E, 2 i \sqrt{E S}]-\left[i . U_{1}(2 i E, 2 i \sqrt{ } E S)\right]\right\}\right.
$$

According to the properties of the Lommel functions

$$
\mathrm{U}_{\mathrm{n}}(\mathrm{z}, \mathrm{w}) \pm \mathrm{i} \mathrm{U}_{\mathrm{n}+1}(\mathrm{z}, \mathrm{w})=\frac{\mathrm{w}^{\mathrm{n}}}{\mathrm{z}^{\mathrm{n}-1}} \int_{0}^{1} \mathrm{~J}_{\mathrm{n}-1}(z t) \exp \left[ \pm \frac{\mathrm{iw}}{2}\left(1-\mathrm{t}^{2}\right)\right] \mathrm{t}^{\mathrm{n}} \cdot d t
$$

We can write the norm of the function $R n$ represented by $N(R n)$ as : 


\section{$N\left(R_{n}\right)=e^{-(E+S)}\left\{2 i E \cdot \int_{0}^{1} J_{-1}(2 i E t) \exp \left[\sqrt{E S}\left(1-t^{2}\right)\right] d t\right\}$ \\ AVERAGE VALUE OF REGISTERED EVENTS}

The average value of the function $\mathrm{Rn}$ is defined by:

$$
\langle n\rangle=\frac{1}{N} \sum_{n=0}^{\infty} n R_{n}
$$

Using the same sequence of procedures developed in the previous section, by calculating the standard, using some properties and, with some simplifications, it is possible to show that:

$$
\langle\mathrm{n}\rangle=\mathrm{E}-\mathrm{S} \cdot\left\{\frac{\mathrm{U}_{2}+\mathrm{i} \mathrm{U}_{1}}{\mathrm{U}_{0}+\mathrm{i} \mathrm{U}_{1}}\right\}
$$

Using the properties of the Lommel functions, presented in the form of integration, it is noticed that On the other hand, we have: $i U_{1}+U_{2}=1 / i\left(i^{2} U_{1}+i U_{2}\right)$,

$$
U_{0}+\mathrm{i} \mathrm{U}_{1}=2 \mathrm{iE} \int_{0}^{1} \mathrm{~J}_{-1}(2 i E t) \cdot \exp \left[-\sqrt{E S}\left(1-t^{2}\right)\right] \mathrm{dt}
$$

this is,

$$
\frac{1}{i}\left(-\mathrm{U}_{1}+\mathrm{i} \mathrm{U}_{2}\right)=\mathrm{i}\left(\mathrm{U}_{1}-\mathrm{i} \mathrm{U}_{2}\right)=-2 \sqrt{\mathrm{ES}} \int_{0}^{1} \mathrm{~J}_{0}(2 i E t) \cdot \exp \left[\sqrt{\mathrm{ES}}\left(1-\mathrm{t}^{2}\right)\right] \cdot \mathrm{t} \mathrm{dt}
$$

So we can write

$$
\langle\mathrm{n}\rangle=\mathrm{E}-\delta . \mathrm{S}
$$

where the factor $\delta$ is given by

$$
\delta=\frac{-2 \sqrt{\mathrm{ES}} \int_{0}^{1} \mathrm{~J}_{0}(2 i E t) \exp \left[\sqrt{\mathrm{ES}}\left(1-t^{2}\right) \cdot \mathrm{t} \mathrm{dt}\right.}{2 i E \int_{0}^{1} \mathrm{~J}_{-1}(2 i E t) \cdot \exp \left[-\sqrt{\mathrm{ES}}\left(1-\mathrm{t}^{2}\right) \mathrm{dt}\right.}
$$

\section{FINAL CONSIDERATIONS}

The average value of the event distribution recorded in $A$ after the bifurcation is not equal to the difference between the mean value of the incident events $(E)$ and the suppressed events $(S)$, that is, (E-S), as we should expect.

In fact, it can be seen that $<\mathrm{n}>=\mathrm{E}-\delta$.S, which can be interpreted as if there is an "interlacing" between the two branches of the bundled (bifurcated) bundle, with the coefficient $\delta$ being the representative parameter of that bundle interaction.

In a way, it is possible to identify a slight similarity between the model proposed in this essay with the studies of Plaga $^{(8)}$ and Monroe ${ }^{(9)}$, which assume the possibility of correlations between distant particles.

\section{BIBLIOGRAPHIC REFERENCES}

1. MERZEBACHER, E. - Quantun Mechanics - Wiley, New York, 1970.

2. BENNETT, C.H. BRASSARD, G. , EKERT, A. Investigación y Ciencia, Spanish edition of Scientific American magazine, n. 195 p. 74-78, 1992.

3. ASPECT, A. and ROGER, G. - Phys. Rev. Lett. V.49, n.2, p.91, 1982.

4. STEWART, I. - Nature, 353, p. 384-385 1991. 
5. RUARK, A. \& DEVOL L. - Phys. Rev. 49, 355, 1936.

6. KINSELLA, I. A., OHANNAIDH, P.B. \& DUNE, A. - American Journal Physics - v. 48, n.2 , p.1972, 1980.

7. BASEIA, B. Brazilian Journal of Physics Teaching, v.17, n.1, 1995

8. PLAGA, RAINER, Foundations of Physics . v.27, n.4, p. 559, 1997.

9. MONROE, C. MEEKHOF, D. M. , KING, B. E Science V. 272, Issue 5265, pp. 1131-1136, 1996.

10. BATEMAN, F. Phi!. Mag. Vol. xx, p. 696, 1910. 